\title{
COMPARATIVE STUDY OF CONCATENATED TURBO CODED AND SPACE-TIME BLOCK CODED AS WELL AS SPACE-TIME TRELLIS CODED OFDM
}

\author{
T. H. Liew, B. J. Choi and L. Hanzo \\ Dept. of ECS., Univ. of Southampton, SO17 1BJ, UK. \\ Tel: +44-703-593 125, Fax: +44-703-593 045 \\ Email: thl97r, lh@ecs.soton.ac.uk http://www-mobile.ecs.soton.ac.uk
}

\begin{abstract}
Space-time block codes provide substantial diversity advantages for multiple transmit antenna systems at a low decoding complexity. In this study, we concatenate both space-time block and trellis codes with Turbo Convolutional (TC) coded Orthogonal Frequency Division Multiplexing (OFDM) for achieving a high coding gain. The associated performance and complexity of the coded OFDM schemes is compared.
\end{abstract}

\section{INTRODUCTION}

In recent years a range of transmit diversity techniques have been proposed, in order to provide diversity gain for MSs by upgrading the BSs, rather than producing millions of more complex diversity-assisted handsets. Only a few years after its invention, space-time coding has been proposed also for employment in the third generation $(3 \mathrm{G})$ mobile communication standards [1]. In [2], Tarokh et al. proposed spacetime trellis coding by jointly designing the channel coding, modulation, transmit diversity and the optional receiver diversity. The proposed space-time trellis codes perform extremely well at the cost of high complexity. In addressing the issue of decoding complexity, Alamouti [3] discovered a remarkable scheme for transmissions using two transmit antennas. A simple decoding algorithm was introduced, which can be generalised to an arbitrary number of receive antennas. This scheme is significantly less complex, than space-time trellis coding using two transmit antennas, although there is a loss in performance.

In this contribution, we concatenate turbo convolutional codes [4] with the space-time block code $\mathbf{G}_{2}$, which is also known as Alamouti's code [3] for improving the performance of the system. The performance of the concatenated space-time block codes and TC codes will then be compared to the performance of space-time trellis codes in the context of Orthogonal Frequency Division Multiplexing (OFDM) [5]. Conventionally, Reed Solomon (RS) codes are also employed in conjunction with space-time trellis codes for improving the performance of the system [6] which will be invoked in our study. The employment of OFDM facilitated space-time coded transmissions over wideband channels.

VTC 2001, Spring, Rhodes, Greece

\section{SYSTEM OVERVIEW}

Figure 1 shows the schematic of our system. At the transmitter, the information source generates random information data bits. The information bits are then encoded by TC codes, RS codes or left uncoded. Only the TC coded bits are channel interleaved and the output bits are then passed to the Space-Time Trellis (STT) or Space-Time Block (STB) encoder of Figure 1. We will investigate the spacetime trellis codes proposed in [2]. The modulation schemes employed are 4-level Phase Shift Keying (4PSK) as well as 8-level Phase Shift Keying (8PSK). On the other hand, from the family of space-time block codes only Alamouti's $\mathbf{G}_{2}$ code is employed in our system, since we have shown in [7] that the best performance is achieved by concatenating the space-time block code $\mathbf{G}_{2}$ with TC codes. Different modulation schemes could be employed [5], such as 16level Quadrature Amplitude Modulation (16QAM) and 64level Quadrature Amplitude Modulation (64QAM). Graymapping of the bits to symbols was applied and this resulted in different protection classes in higher-order modulation schemes [5]. The mapping of the data bits and parity bits of the TC encoder was chosen such that it yielded the best achievable performance along with the application of the random separation channel interleaver of [8]. The output of the space-time encoder was then OFDM [5] modulated with the aid of the Inverse Fast Fourier Transform (IFFT) blocks of Figure 1 and transmitted by the corresponding antenna. The number of transmit antennas was fixed to two, while the number of receive antennas constituted a design parameter. Dispersive wideband channels were used and the associated channels' profiles will be discussed at a later stage.

At the receiver the signal of each receive antenna is OFDM demodulated. The demodulated signals of the receiver antennas are then fed to the space-time trellis or space-time block decoder. The space-time decoders apply the Logarithmic Maximum A-Posteriori (Log-MAP) $[9,10]$ decoding algorithms for providing soft outputs for the channel decoders. If no channel codecs are employed in the system, the space-time decoders apply the Viterbi Algorithm (VA) [2], which gives a slightly lower performance compared to the MAP decoder, but at a lower complexity. The decoded bits are finally passed to the sink, as seen in Figure 1, for the calculation of the Bit Error Rate (BER) or Frame Error Rate (FER). 


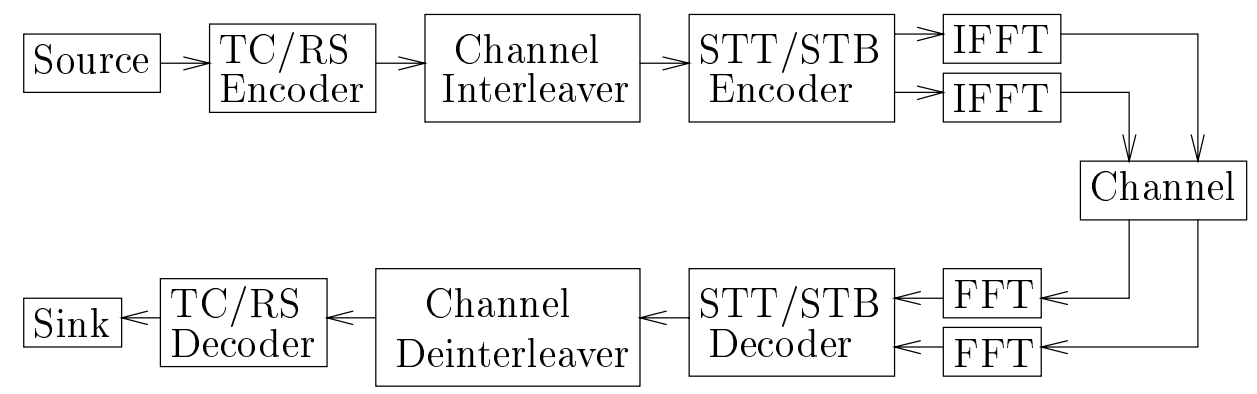

Figure 1: System overview.

\section{SPACE-TIME AND CHANNEL CODEC PARAMETERS}

In Figure 1, we have given an overview of the proposed system. In this section, we present the parameters of the space-time trellis codes and the channel codecs employed in the proposed system. We will employ the set of various space-time trellis codes proposed in [2]. The associated space-time trellis coding parameters are summarised in Table 1 .

\begin{tabular}{|c|c|c|c|c|}
\hline $\begin{array}{c}\text { Modulation } \\
\text { scheme }\end{array}$ & BPS & $\begin{array}{l}\text { Decoding } \\
\text { algorithm }\end{array}$ & $\begin{array}{l}\text { No. of } \\
\text { trellis } \\
\text { states }\end{array}$ & $\begin{array}{c}\text { No. of } \\
\text { termination } \\
\text { symbols }\end{array}$ \\
\hline \multirow[t]{4}{*}{ 4PSK } & \multirow[t]{4}{*}{2} & \multirow[t]{4}{*}{ VA } & 4 & 1 \\
\hline & & & 8 & 2 \\
\hline & & & 16 & 2 \\
\hline & & & 32 & 3 \\
\hline \multirow[t]{3}{*}{ 8PSK } & \multirow[t]{3}{*}{3} & \multirow[t]{3}{*}{$\overline{\mathrm{VA}}$} & 8 & 1 \\
\hline & & & 16 & 2 \\
\hline & & & 32 & 2 \\
\hline
\end{tabular}

Table 1: Parameters of the space-time trellis codes [2]

In this treatise, we will concentrate on using the simple half-rate $\mathrm{TC}(2,1,3)$ code. Its associated parameters are shown in Table 2. As seen in Table 3, different modulation

\begin{tabular}{|c|c|c|c|c|}
\hline Code & $\begin{array}{c}\text { Octal } \\
\text { generator } \\
\text { polynomial }\end{array}$ & $\begin{array}{c}\text { No. } \\
\text { of } \\
\text { states }\end{array}$ & $\begin{array}{c}\text { Decoding } \\
\text { algorithm }\end{array}$ & $\begin{array}{c}\text { No. } \\
\text { of } \\
\text { iterations }\end{array}$ \\
\hline $\mathrm{TC}(2,1,3)$ & 7,5 & 4 & Log-MAP & 8 \\
\hline
\end{tabular}

Table 2: Parameters of the TC $(2,1,3)$ codec.

schemes are employed in conjunction with the concatenated space-time block code $\mathbf{G}_{2}$ and the TC $(2,1,3)$ code. Since the half-rate TC $(2,1,3)$ code is employed, higher-order modulation schemes such as 16QAM and 64QAM were chosen, so that the Bits Per Symbol (BPS) throughput of the system remained the same as that of the system employing the space-time trellis codes without additional channel coding. It is widely recognised that the performance of $\mathrm{TC}$ codes improves upon increasing the turbo interleaver size and near-Shannonian performance can be achieved using large interleaver sizes exceeding 10,000 bits. However, this performance gain is achieved at the cost of high latency, which is impractical for a delay-sensitive real-time system. On the other hand, space-time trellis codes offer impressive coding gains [2] at low latency. The decoding of the spacetime trellis codes is carried out on a transmission burst-byburst basis. In order to make a fair comparison between the systems investigated, the turbo interleaver size was chosen such that all the coded bits of a specific interleaved block were hosted by one transmission burst. This enables burstby-burst turbo decoding at the receiver. In Table 3 , we

\begin{tabular}{|l|c|c|c|c|c|}
\hline \multirow{2}{*}{ Code } & $\begin{array}{c}\text { Code } \\
\text { Rate } \\
R\end{array}$ & $\begin{array}{c}\text { Modu- } \\
\text { lation } \\
\text { Mode }\end{array}$ & BPS & $\begin{array}{c}\text { Random } \\
\text { turbo } \\
\text { interleaver } \\
\text { depth }\end{array}$ & $\begin{array}{c}\text { Random } \\
\text { separation } \\
\text { interleaver } \\
\text { depth }\end{array}$ \\
\hline TC $(2,1,3)$ & 0.50 & 16QAM & 2 & 256 & 512 \\
\cline { 3 - 6 } & & $64 \mathrm{QAM}$ & 3 & 384 & 768 \\
\hline
\end{tabular}

Table 3: Additional system parameters associated with the $\mathrm{TC}(2,1,3)$ code.

summarised the modulation schemes and interleaver sizes used in the proposed system. Again, the random separation based channel interleaver of [8] was used. The mapping of the data bits and parity bits into different protection classes of the higher-order modulation scheme [5] was carried out such that the best possible performance was attained [8].

\begin{tabular}{|l|c|c|c|}
\hline Code & $\begin{array}{c}\text { Galois } \\
\text { Field }\end{array}$ & $\begin{array}{c}\text { Code } \\
\text { rate }\end{array}$ & $\begin{array}{c}\text { Correctable } \\
\text { symbol errors }\end{array}$ \\
\hline $\mathrm{RS}(63,42)$ & $2^{6}$ & 0.67 & 10 \\
\hline
\end{tabular}

Table 4: The coding parameters of the Reed-Solomon codes employed.

Following the rationale of [6], Reed-Solomon codes were employed in conjunction with the space-time trellis codes. Hard decision RS decoding was utilised and the coding parameters of the Reed-Solomon codes employed are summarised in Table 4.

\subsection{Complexity Issues}

In this section, we will address the implementational complexity issues of the proposed system. We will however focus mainly on the relative complexity of the proposed systems, rather than attempting to quantify their exact complexity. In order to simplify our comparative study, several assumptions were stipulated. In our simplified approach, the estimated complexity of the system is deemed to depend only on that of the space-time trellis decoder and turbo decoder. In other words, the complexity associated with the modulator, demodulator, space-time block encoder and decoder as well as that of the space-time trellis encoder and turbo encoder are assumed to be insignificant compared to the complexity of space-time trellis decoder and turbo decoder.

In [7], we have derived our complexity estimates for the TC decoder and the reader is referred to [7] for further details. The estimated complexity of the TC decoder is 


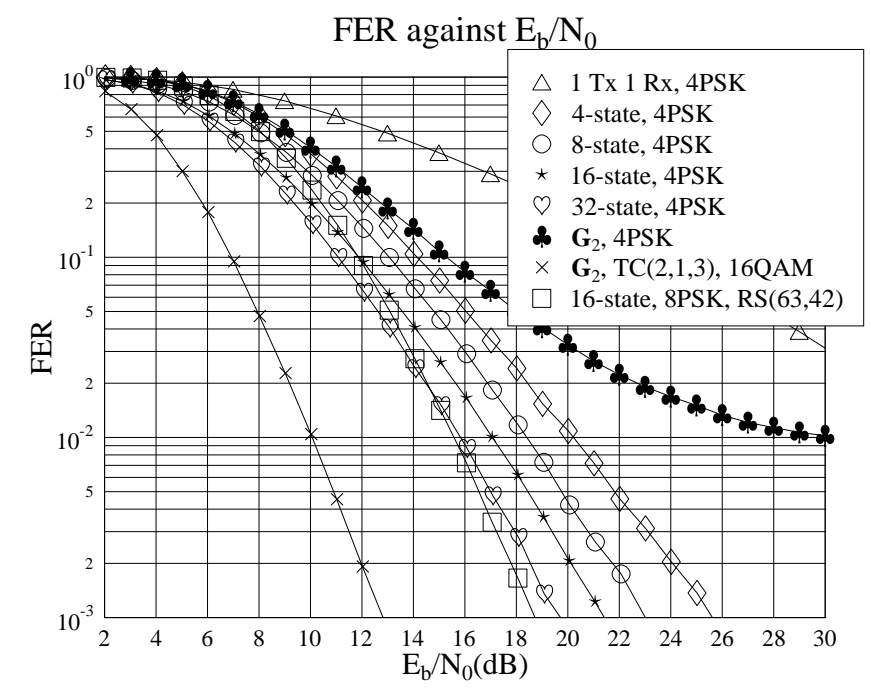

Figure 2: FER performance comparison between various 4PSK space-time trellis codes and the space-time block code $\mathbf{G}_{2}$ concatenated with the $\mathrm{TC}(2,1,3)$ code and the 16 -state $8 \mathrm{PSK}$ spacetime trellis code concatenated with the $\mathrm{RS}(63,42)$ code over $\mathrm{GF}\left(2^{6}\right)$ using one receiver and the 128 -subcarrier OFDM modem over a channel having a CIR characterised by two equalpower rays separated by a delay spread of $5 \mu \mathrm{s}$. The maximum Doppler frequency was $200 \mathrm{~Hz}$. The effective throughput was 2 BPS and the coding parameters are shown in Tables 1, 2, 3 and 4.

assumed to depend purely on the number of trellis transitions per information data bit and this simple estimated complexity measure was used in [7] as the basis of our comparisons. Here, we adopt the same approach and quantify the estimated complexity of the space-time trellis decoder also on the basis of the number of trellis transitions per information data bit.

From the state diagrams shown in [2], we can see that the number of trellis transitions leaving each state is equivalent to $2^{B P S}$, where, again, $B P S$ denotes the number of transmitted bits per modulation symbol. Since the number of information bits is equal to BPS, we can approximate the complexity of the space-time trellis decoder as:

$$
\operatorname{comp}\{\mathrm{STT}\}=\frac{2^{B P S} \times \text { No. of States }}{B P S}
$$

\section{SIMULATION RESULTS}

In this section, we provide simulation results for space-time coded OFDM schemes using 128 subcarriers. Each OFDM symbol has a symbol duration of $160 \mu s$ and a cyclic prefix of $40 \mu s$ duration. In these simulations, the Jakes model was adapted for modelling the fading channels [11]. We assume an equal-power two-path Channel Impulse Response (CIR), where the CIR taps are separated by a delay spread of $5 \mu \mathrm{s}$. Furthermore, in our simulations we also present results over two-ray channels separated by various delay spreads, up to $40 \mu \mathrm{s}$. The maximum Doppler frequency was $200 \mathrm{~Hz}$. All multipath components undergo independent Rayleigh fading and the receiver has a perfect knowledge of the CIR.

\subsection{Systems Having A Throughput Of 2 BPS}

In Figure 2, we show our FER performance comparison between the various 2 BPS effective throughput schemes, namely the 4PSK space-time trellis codes and the spacetime block code $\mathbf{G}_{2}$ concatenated with the $\mathrm{TC}(2,1,3)$ code and the 16 -state 8 PSK space-time trellis code concatenated with the $\operatorname{RS}(63,42)$ code over $\operatorname{GF}\left(2^{6}\right)$ using one receiver and the 128-subcarrier OFDM modem. More explicitly, the $\mathrm{TC}(2,1,3)$ code is a half-rate code and hence 16QAM was employed for absorbing its parity bits, in order to support the same 2 BPS effective throughput, as the 4PSK spacetime trellis codes using no channel codes. Similarly, the $\mathrm{RS}(63,42)$ code has a coding rate of about two-third and hence the 16-state 8PSK space-time trellis code was employed for maintaining a similar effective throughput. We can clearly see that at $\mathrm{FER}=10^{-3}$ the performance of the $\mathrm{TC}(2,1,3) / \mathbf{G}_{2}$ concatenated scheme is at least $7 \mathrm{~dB}$ better, than that of the 4PSK space-time trellis codes. The concatenation of the 16-state 8PSK space-time trellis code and the $\mathrm{RS}(63,42)$ code improves the performance and outperforms the 4PSK space-time trellis codes. However, its performance is still inferior to the $\mathrm{TC}(2,1,3) / \mathbf{G}_{2}$ scheme.

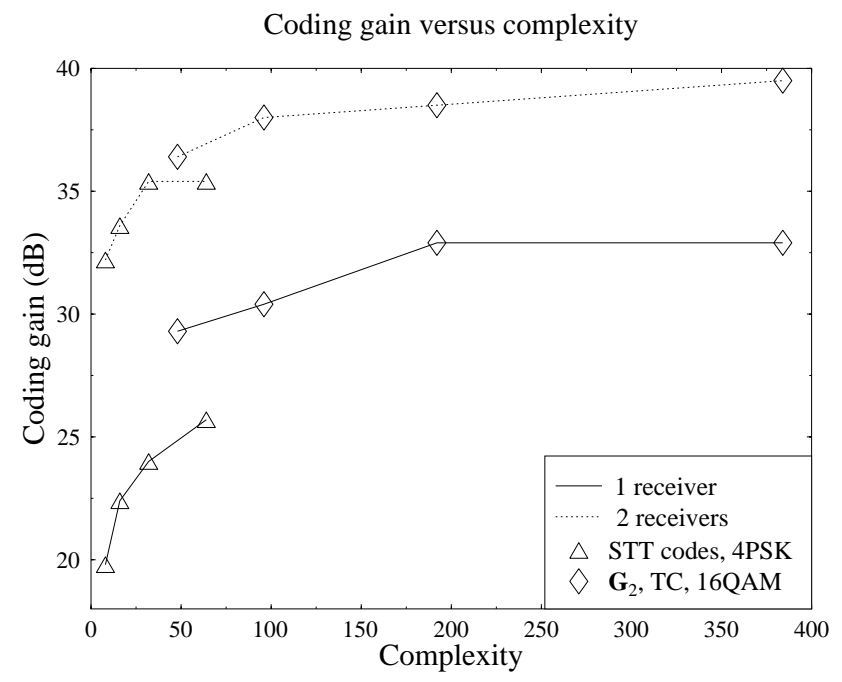

Figure 3: Coding gain versus estimated complexity for the various 4PSK space-time trellis codes and the space-time block code $\mathbf{G}_{2}$ concatenated with the $\mathrm{TC}(2,1,3)$ code using one as well as two receivers and the 128 -subcarrier OFDM modem over a channel having a CIR characterised by two equal-power rays separated by a delay spread of $5 \mu \mathrm{s}$. The maximum Doppler frequency was $200 \mathrm{~Hz}$. The effective throughput was 2 BPS and the coding parameters are shown in Tables 1, 2 and 3.

In Section 3.1 and [7], we provided the complexity estimates of the TC decoders and space-time trellis decoders, respectively. By employing Equation 1 and the corresponding equations in [7], we compare the performance of the proposed schemes by considering their approximate complexity. Our performance comparison of the various schemes in Figure 3 was carried out on the basis of the coding gain defined as the $E_{b} / N_{0}$ difference, expressed in decibels $(\mathrm{dB})$, at $\mathrm{FER}=10^{-3}$ between the proposed schemes and the uncoded single-transmitter, single-receiver system having the same effective throughput of 2 BPS. Specifically, in 
Figure 3, we show our coding gain versus estimated complexity comparison for the various 4PSK space-time trellis codes and the space-time block code $\mathbf{G}_{2}$ concatenated with the $\mathrm{TC}(2,1,3)$ code using one as well as two receivers. The 128-subcarrier OFDM modem transmitted over the channel having a CIR of two equal-power rays separated by a delay spread of $5 \mu \mathrm{s}$ and a maximum Doppler frequency of $200 \mathrm{~Hz}$. The estimated complexity of the space-time trellis codes was increased by increasing the number of trellis states. By contrast, the estimated complexity of the $\mathrm{TC}(2,1,3)$ code was increased by increasing the number of turbo iterations. Therefore, the coding gain of the concatenated $\mathbf{G}_{2} / \mathrm{TC}(2,1,3)$ scheme using one, two, four and eight iterations is shown in Figure 3. It can be seen that the concatenated scheme outperforms the space-time trellis codes using no channel coding, even though the number of turbo iterations was only one. Moreover, the improvement in coding gain was obtained at an estimated complexity comparable to that of the 32-state 4PSK space-time trellis code using no channel coding. From the figure we can also see that the performance gain of the concatenated $\mathbf{G}_{2} / \mathrm{TC}(2,1,3)$ channel coded scheme over the space-time trellis codes becomes lower, when the number of receivers is increased to two.

\subsection{Systems Having A Throughput Of 3 BPS}

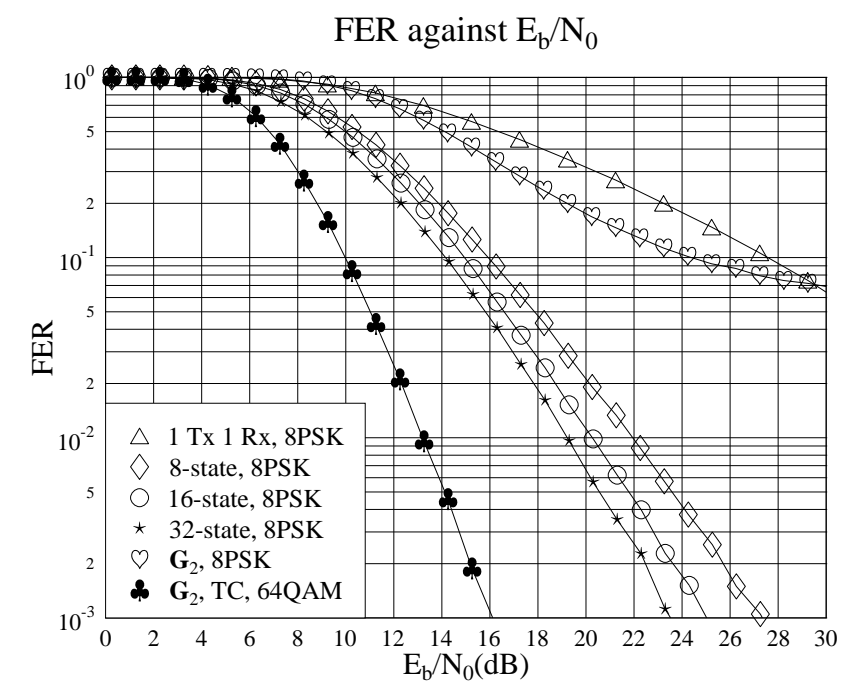

Figure 4: FER performance comparison between various 8PSK space-time trellis codes and the space-time block code $\mathbf{G}_{2}$ concatenated with the $\mathrm{TC}(2,1,3)$ code using one receiver and the 128-subcarrier OFDM modem over a channel having a CIR characterised by two equal-power rays separated by a delay spread of $5 \mu \mathrm{s}$. The maximum Doppler frequency was $200 \mathrm{~Hz}$. The effective throughput was 3 BPS and the coding parameters are shown in Tables 1,2 and 3.

In Figure 4, we show our FER performance comparison between the various 8PSK space-time trellis codes of Table 1 and space-time block code $\mathbf{G}_{2}$ concatenated with the $\mathrm{TC}(2,1,3)$ code using one receiver and the 128 -subcarrier OFDM modem. Since the $\mathrm{TC}(2,1,3)$ scheme is a half-rate code, 64QAM was employed in order to maintain the same 3 BPS effective throughput, as that of the 8PSK spacetime trellis codes using no channel coding. We can clearly see that at $\mathrm{FER}=10^{-3}$ the performance of the concatenated channel coded scheme is at least $7 \mathrm{~dB}$ better in terms of the required $E_{b} / N_{0}$, than that of the space-time trellis codes. The performance of the space-time block code $\mathbf{G}_{2}$ without the concatenated $\mathrm{TC}(2,1,3)$ code is also shown in the figure. In Table 3, we can see that although there is an increase in the turbo interleaver size, due to employing a higher-order modulation scheme, nonetheless, no performance gain is observed for the concatenated TC(2, 1,3)- $\mathbf{G}_{2}$ scheme over the space-time trellis codes using no channel coding. We speculate that this is because the potential gain due to the increased interleaver size has been offset by the increased BER of the more vulnerable 64QAM scheme.

\section{THE EFFECT OF DELAY SPREAD}

In this section, we will study how the variation of the delay spread between the two paths of the channel affects the system's performance. Figure 5 shows the baseband represen-

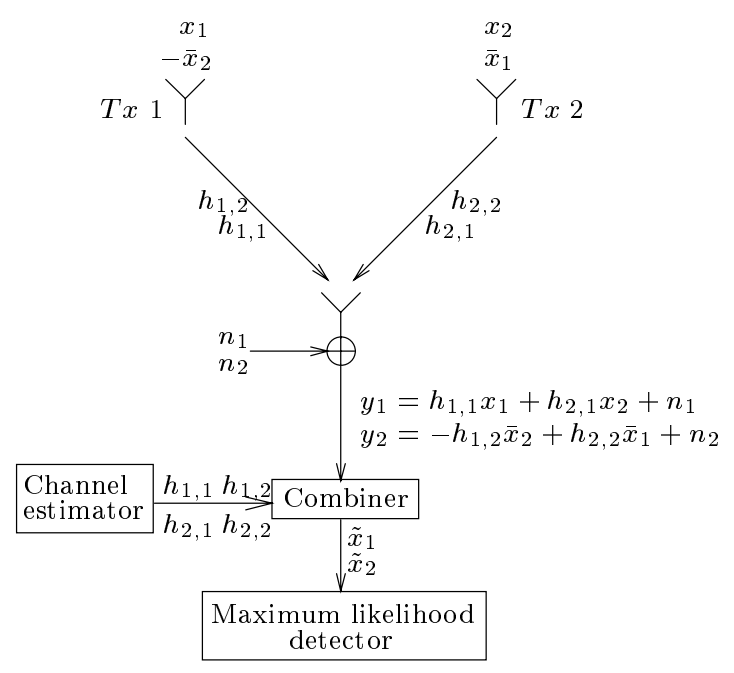

Figure 5: Baseband representation of the simple twintransmitter space-time block code $\mathbf{G}_{2}$ using one receiver over varying fading conditions.

tation of the simple twin-transmitter space-time block code $\mathbf{G}_{2}$ using one receiver. The two transmission instants of the $\mathbf{G}_{2}$ code can are no longer assumed to be associated with the same complex transfer function values. This is because the two original time-slots of the $\mathbf{G}_{2}$ code are now mapped to two adjacent OFDM subcarriers, which may have different complex transfer factors due to the dispersive channel's frequency selective fading. Hence at the receiver, we have

$$
\begin{aligned}
& y_{1}=h_{1,1} x_{1}+h_{2,1} x_{2}+n_{1} \\
& y_{2}=-h_{1,2} \bar{x}_{2}+h_{2,2} \bar{x}_{1}+n_{2},
\end{aligned}
$$

where $y_{1}$ is the first received signal and $y_{2}$ is the second. Both signals $y_{1}$ and $y_{2}$ are passed to the combiner in order to extract the signals $x_{1}$ and $x_{2}$. Aided by the channel estimator, which in this example provides perfect estimation of the CIR, the combiner performs the required simple signal processing in order to separate the signals $x_{1}$ and $x_{2}$. Specifically, in order to extract the signal $x_{1}$, we combine 
the signals $y_{1}$ and $y_{2}$ as follows:

$$
\begin{aligned}
\tilde{x}_{1}= & \bar{h}_{1,1} y_{1}+h_{2,2} \bar{y}_{2} \\
= & \left(\left|h_{1,1}\right|^{2}+\left|h_{2,2}\right|^{2}\right) x_{1}+ \\
& \left(\bar{h}_{1,1} h_{2,1}-h_{2,2} \bar{h}_{1,2}\right) x_{2}+\bar{h}_{1,1} n_{1}+h_{2,2} \bar{n}_{2}
\end{aligned}
$$

In contrast to the prefect cancellation scenario of $h_{1,1}=$ $h_{1,2}$ and $h_{2,1}=h_{2,2}$, we can see from Equation 4 that the signals $x_{1}$ and $x_{2}$ now interfere with each other. Hence we can no longer cancel the cross-coupling of signals $x_{2}$ and $x_{1}$ in Equation 4, unless the channel transfer factors of the adjacent OFDM subcarriers satisfy the condition of $h_{1,1}=h_{1,2}$ and $h_{2,1}=h_{2,2}$.

At high SNRs, the noise power is insignificant compared to the transmitted power of the signals $x_{1}$ and $x_{2}$. Therefore, we can ignore the noise terms in Equation 4. However, the interference signals' power increases, as we increase the transmission power. Assuming that both the signals $x_{1}$ and $x_{2}$ have an equivalent signal power, we can then express the signal to interference ratio (SIR) for signal $x_{1}$ as:

$$
S I R=\frac{\left|h_{1,1}\right|^{2}+\left|h_{2,2}\right|^{2}}{\bar{h}_{1,1} h_{2,1}-h_{2,2} \bar{h}_{1,2}},
$$

and similarly for signal $x_{2}$ as:

$$
S I R=\frac{\left|h_{2,1}\right|^{2}+\left|h_{1,2}\right|^{2}}{\bar{h}_{2,1} h_{1,1}-h_{1,2} \bar{h}_{2,2}} .
$$

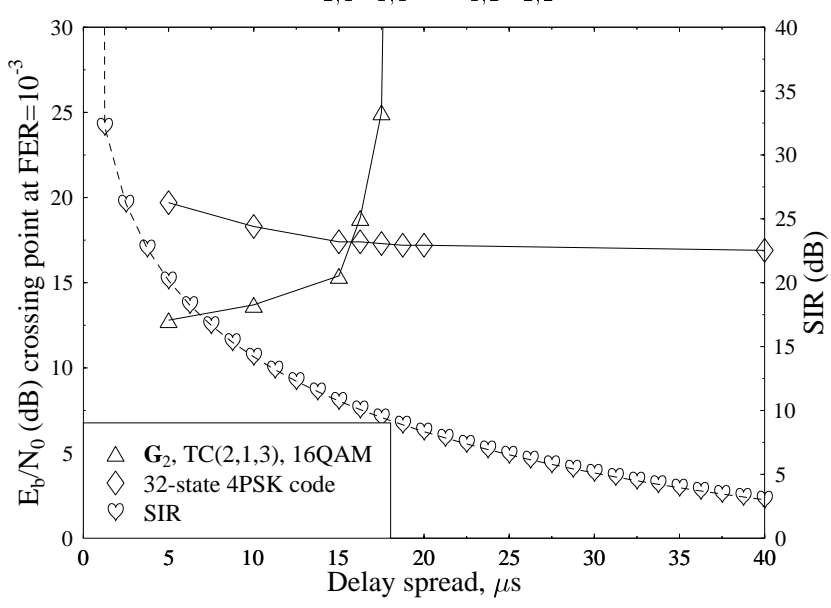

Figure 6: The $E_{b} / N_{0}$ values required for maintaining $\mathrm{FER}=10^{-3}$ versus delay spreads for the 32 -state $4 \mathrm{PSK}$ spacetime trellis code and for the space-time block code $\mathbf{G}_{2}$ concatenated with the TC $(2,1,3)$ code using one receiver and the 128subcarrier OFDM modem. The CIR exhibited two equal-power rays separated by various delay spreads and a maximum Doppler frequency of $200 \mathrm{~Hz}$. The effective throughput was 2 BPS and the coding parameters are shown in Tables 1, 2 and 3 . The SIR of various delay spreads are shown as well.

We show in Figure 6 the $E_{b} / N_{0}$ value required for maintaining $\mathrm{FER}=10^{-3}$ versus the delay spread for the 32 -state 4PSK space-time trellis code and for the space-time block code $\mathbf{G}_{2}$ concatenated with the $\mathrm{TC}(2,1,3)$ code using one receiver and the 128-subcarrier OFDM modem. The CIR exhibited two equal-power rays separated by various delay spreads. The maximum Doppler frequency was 200 Hz. The $S I R$ associated with the various delay spreads was obtained using computer simulations and the associated $S I R$ values are also shown in Figure 6, denoted by the hearts and scaled on the right-hand axis. As we have expected, the calculated $S I R$ decreases upon increasing the delay spread. We can see in the figure that the performance of the 32-state 4PSK space-time trellis code does not vary significantly with the delay spread. On the other hand, the performance of the concatenated $\mathbf{G}_{2} / \mathrm{TC}(2,1,3)$ scheme suffers severe degradation, when the delay spread is in excess of $15 \mu \mathrm{s}$, as indicated by the near-vertical curve of Figure 6 marked by triangles. If we relate this curve to the SIR curve marked by the hearts, we can see on the right-hand side scale of the figure that the $S I R$ is approximately $10 \mathrm{~dB}$.

In conclusion, the $\mathbf{G}_{2} / \mathrm{TC}(2,1,3)$ OFDM scheme significantly outperformed the STTC OFDM schemes at the throughputs of 2 and 3 BPS, when stipulating the same system complexity at a CIR dispersion of $5 \mu \mathrm{s}$. However, the $S I R$ of the concatenated $\mathbf{G}_{2} / \mathrm{TC}(2,1,3)$ scheme has to be more than $10 \mathrm{~dB}$, in order for it to outperform the spacetime trellis codes using no channel coding over strongly dispersive channels. Our future research will be considering adaptive space-time coded OFDM modems.

\section{REFERENCES}

[1] P. Chaudhury, "The 3GPP Proposal for IMT-2000", IEEE Communications Magazine, Vol. 37, no. 12, pp. 72-81, December 1999

[2] V. Tarokh, N. Seshadri and A. R. Calderbank, "Space-Time Codes for High Data Rate Wireless Communication: Performance Criterion and Code Construction", IEEE Transactions on Information Theory, Vol. 44, no. 2 pp. 744-765, March 1998.

[3] S. M. Alamouti, "A Simple Transmit Diversity Technique for Wireless Communications", IEEE JSAC, pp. 1451-1458, October 1998.

[4] C. Berrou and A. Glavieux, "Near Optimum Error Correcting Coding and Decoding: Turbo-Codes", IEEE Transactions on Communications, Vol. 44, no. 10, pp. 1261-1271, October 1996.

[5] L. Hanzo, W. Webb and T. Keller, "Single- and Multicarrier Quadrature Amplitude Modulation", John Wiley \& Sons, Ltd, Wessex, England, ISBN 0471492396.

[6] A. Naguib, N. Seshadri and A. Calderbank, "Increasing Data Rate Over Wireless Channels", IEEE Signal Processing Magazine, Vol. 17, no. 3, pp. 76-92, May 2000.

[7] T. Liew, J. Pliquett, B. Yeap, L-L. Yang and L. Hanzo, "Concatenated Space Time Block Codes and TCM, Turbo TCM, Convolutional as well as Turbo Codes", Globecom 2000, San Francisco, USA, pp 2292-2301, 27 Nov -1 Dec 2000 .

[8] T. Liew, J. Pliquett, B. Yeap, L-L. Yang and L. Hanzo, "Comparative Study of Space Time Block Codes and Various Concatenated Turbo Coding Schemes", PIMRC 2000, London, UK, pp 741-745, 18-21 Sept 2000.

[9] P. Robertson, E. Villebrun and P. Hoeher, "A Comparison of Optimal and Sub-Optimal MAP Decoding Algorithms Operating in the Log Domain", Proceedings of the International Conference on Communications, pp 1009-1013, June 1995.

[10] G. Bauch, "Concatenation of Space-Time Block Codes and Turbo-TCM", Proc IEEE ICC, Vancouver, Canada pp .1202-1206, June 1999.

[11] W. C. Jakes, "Microwave Mobile Communications", IEEE Press, Piscataway NJ, 1993. 